\title{
Head posture effects in prism adaptation during hallway exposure
}

\author{
GORDON M. REDDING \\ Illinois State University, Normal, Illinois \\ and \\ BENJAMIN WALLACE \\ Cleveland State University, Cleveland, Ohio
}

\begin{abstract}
Different groups of subjects walked nearer the left or right side of a hallway while looking through prisms that displaced the visual field in either the left or right direction. As predicted from the head posture required to view the apparently displaced hallway, adaptive pre-post change in felt head position was greater when head turning during exposure was expected to be greater (i.e., when the subject was walking on the prism base side of the hall). This finding supports a postural basis, but not a discordance basis, for change in felt head position. Visual adaptation was not explained either by the head position results or by the eye-in-head posture required to view the apparently displaced hallway, and was attributed to recalibration processes based on discordant input from other sensorimotor systems. Proprioceptive adaptation in the right hand was small and similar to the head position results, except for the group that experienced rightward displacement and walked near the right wall. The substantial right-hand proprioceptive adaptation in this group was attributed to discordance between visual and proprioceptive systems when subjects visually anticipated an encounter between their arm or shoulder and the wall.
\end{abstract}

When people first experience an optical transformation such as lateral displacement of the visual field, they show obvious behavioral difficulty. For instance, they tend to be hesitant and to stumble when walking a familiar path, such as a hallway. However, these obvious errors soon disappear, usually within a few minutes, and the person appears to have adapted to the distorted visual stimulation. Experimental tests have demonstrated that this adaptation includes perceptual changes in both vision and proprioception (e.g., Hay \& Pick, 1966; Redding \& Wallace, 1976, 1978; Wallace \& Redding, 1979; Wilkinson, 1971). The present study extends our attempt to understand the variable nature of such perceptual adaptation (for a review see Redding, Clark, \& Wallace, 1985).

The usual measure of visual shift (VS) requires remote adjustment of a visual target to appear straight ahead of the nose before and after prism exposure. This measure is logically sensitive to any change in perceived visual location involving the eye-in-head system. For instance, VS may be due to a change in retinal local sign or in registered eye position (e.g., Craske \& Crawshaw, 1974), and a change in eye position may arise either from recalibration of the eye position sense to reduce the discordance produced by the prisms between vision and other perceptual modalities (e.g., Craske \& Crawshaw, 1978) or from muscle potentiation aftereffects of asymmetric eye posture

\footnotetext{
Special thanks are due Jeff Imig and Dennis Householder for their unflagging encouragement and indispensible assistance in developing the test apparatus used in this study. Correspondence should be addressed to Gordon M. Redding, Department of Psychology, llinois State University, Normal, IL 61761 .
}

induced by scanning the displaced visual array (e.g., Ebenholtz, 1976). ${ }^{1}$

The usual measure of proprioceptive shift (PS) requires pointing straight ahead of the nose without sight of the hand before and after prism exposure. This measure is logically sensitive to any change in proprioception between the head and hand. For instance, PS may be due to change in felt hand or head position or both (e.g., Redding \& Wallace, 1987), and a change in head position may arise either from recalibration of the head position sense or from aftereffects of asymmetric head posture induced by exploration of the displaced visual space (e.g., Ebenholtz, 1976; Howard \& Anstis, 1974).

Although changes based on either recalibration or postural aftereffects are "perceptual" in the sense that they entail adaptive perceptual response to the displacement, it is important to identify these two kinds of adaptive components, especially the contribution of head posture (Redding \& Wallace, 1987, 1988). Moreover, depending upon head posture, the eyes may or may not be asymmetrically exercised during prism exposure, and head posture may therefore contribute indirectly to VS as well as to PS.

Change in felt position of the head has long been thought to be a possible consequence of exposure to prismatic displacement (e.g., Cohen, 1967; Hamilton, 1964; Harris, 1965), but most of the evidence for such effects is indirect (Welch, 1978, pp. 63-64). Kohler $(1964$, p. 38) reported that prism-wearing subjects were unaware of turned head postures, Ebenholtz (1976) reported aftereffects of turned head postures, and Redding and Wal- 
lace (1988) found small head shift (HS) following hand exposure to displacement, but there have been no quantitative measures of HS following hallway exposure. In the present experiment we attempted to test for head posture effects. The following exposition of the test is rather lengthy, since the logic has not previously been fully developed (but for a preliminary statement, see Redding \& Wallace, 1987).

When subjects first put on laterally displacing prisms in hallway exposure, the visual appearance of the world is as it would be if the head were turned in the base direction by the amount of the displacement. That is, the hallway appears to angle off in the displacement direction as it would if the head were turned in the base direction. Thus, the visual information indicates that the head is turned in the base direction, even though it remains straight on the shoulders. This discordance between visual information about head position and felt head position may form the basis for a more enduring perceptual recalibration, such that the head comes to feel turned in the base direction when it is straight on the shoulders even without the discordance-producing visual input (i.e., an aftereffect). ${ }^{2}$ The predicted HS is then in the direction of the displacement when subjects are asked, without vision, to turn the head to be straight on their shoulders.

Note that if the head is kept straight during exposure, the eyes must turn in the displacement direction to fixate in the direction of locomotion (as specified by the optical flow pattern and hallway structure). Thus, eye position is asymmetrical and there is a basis for eye-muscle potentiation aftereffects (e.g., Ebenholtz, 1976) to appear on the VS test.

However, the head is not usually kept perfectly straight. There is a (sometimes slight) tendency to turn the head in the displacement direction (Kohler, 1964, p. 38). Such head turning is largest when subjects are near the wall on the base side, because all they see without turning their head may be the near wall. In order to fully see the hall, subjects may need to turn their head by a larger amount than when the nearest wall is on the displacement side. This head turning tends to center the structured array and direction of locomotion in the available visual field, thereby better enabling locomotion. In this situation, visual information indicates a straight head, whereas it is actually turned in the displacement direction. Thus, again, the discordance between seen and felt head position may form the basis for a more enduring change in felt head position, such that the head comes to feel straight on the shoulders when it is turned in the displacement direction even without the visual input (i.e., an aftereffect). Again, the predicted HS is in the direction of the displacement (i.e., when asked to position the head straight on the shoulders without vision, a subject should turn it in the displacement direction). Note, however, that this need not be a true perceptual recalibration effect (which is presumed to be based directly on discordant sensory inputs), but may be a purely local change based on a departure from the usual perceptual significance associated with input from position receptors (Ebenholtz, 1976; Howard \& Anstis, 1974).

Again, note that to the extent that the head is turned in the displacement direction, the eyes will be symmetrically positioned in the head to fixate the center of the structured array and the direction of locomotion. Thus, head turning in the direction of displacement reduces any eyemuscle potentiation component of VS. On the other hand, such head turning should increase postural aftereffects in the neck and should increase this component of a PS test that is referenced to the head (e.g., pointing straight ahead of the nose without vision).

Now, the logic of the specific predictions shown in Table 1 can be developed. The exposure conditions are produced by a factorial combination of whether subjects are instructed to walk near the right or left side of the hall and whether the displacement direction is rightward (prism base left) or leftward (base right).

When subjects are instructed to walk near the displacement side of the hall, they should tend to keep the head straight (less head turning in the displacement direction), but the head should look turned opposite the displacement direction. That is, the local head position signal in the head-trunk system veridically indicates the straight head position, but information from the remote visual system indicates a head position turned in the base direction. Thus, any postural aftereffect component of the HS test should be small, but the discordance between felt and seen head position may provide the basis for perceptual recalibration of head position.

In these conditions, the eyes must turn in the displacement direction for the subject to see straight down the hallway. Such asymmetric eye position may produce postural aftereffects that would appear on the VS test, but note that there is also a discordance basis for a change in registered eye position. The eyes are actually turned in the displacement direction to see straight ahead, but the head is actually straight. This discordance between eyehead and head-trunk systems may produce recalibration of registered eye position.

When subjects are instructed to walk on the base side of the hall, they should tend to turn the head in the displacement direction (to see the hall), but the visual information would indicate that the head is straight. Thus, the HS test may include both postural and recalibration aftereffect components and HS may be larger than in the preceding case.

To the extent that the head is turned, the eyes are straight in the head and there is no basis for eye posture aftereffects, but the possibility remains of discordancebased change in registered eye position. That is, the eyes are straight in the head but the head is turned in the displacement direction, indicating that the eyes are turned in the base direction. Thus, a straight eye position may come to signify a position in the base direction, and for the eyes to be registered as straight in the absence of the 
Table 1

Directional Predictions for Combinations of Displacement Direction and the Wall Nearest Which Subjects Were Instructed to Walk During Hallway Exposure

\begin{tabular}{|c|c|c|c|c|}
\hline & \multicolumn{2}{|c|}{$\begin{array}{l}\text { Rightward Displacement } \\
\text { (Base Left Prisms) }\end{array}$} & \multicolumn{2}{|c|}{$\begin{array}{l}\text { Leftward Displacement } \\
\text { (Base Right Prisms) }\end{array}$} \\
\hline & Right Wall & Left Wall & Right Wall & Left Wall \\
\hline \multicolumn{5}{|c|}{ Position Signals* } \\
\hline $\begin{array}{l}\text { Head-Trun } \\
\text { Local } \\
\text { Remote }\end{array}$ & $\begin{array}{l}\mathbf{S} \\
\mathrm{L}\end{array}$ & $\begin{array}{l}\mathbf{R} \\
\mathrm{S}\end{array}$ & $\begin{array}{l}\mathbf{L} \\
\mathbf{S}\end{array}$ & $\begin{array}{l}\mathbf{S} \\
\mathbf{R}\end{array}$ \\
\hline $\begin{array}{l}\text { Eye-Head } \\
\text { Local } \\
\text { Remote }\end{array}$ & $\begin{array}{l}\mathbf{R} \\
\mathbf{S}\end{array}$ & $\begin{array}{l}S \\
\text { L }\end{array}$ & $\begin{array}{l}\mathbf{S} \\
\mathbf{R}\end{array}$ & $\begin{array}{l}\text { L } \\
\text { S }\end{array}$ \\
\hline
\end{tabular}

Aftereffects $\dagger$

$\begin{array}{lllll}\text { Head Shift } & & & & \\ \quad \text { Postural } & \mathbf{0} & \mathbf{R} & \text { L } & \mathbf{0} \\ \text { Discordance } & \mathbf{R} & \mathbf{R} & \text { L } & \text { L } \\ \text { Visual Shift } & & & & \\ \text { Postural } & \mathbf{R} & \mathbf{0} & \mathbf{0} & \text { L } \\ \text { Discordance } & \mathbf{R} & \mathbf{R} & \text { L } & \text { L }\end{array}$

Note $-\mathrm{L}=$ left, $\mathrm{R}=$ right, $\mathrm{S}=$ straight, $0=$ no change. ${ }^{*}$ Type of position signal expected in the head-trunk and eye-head systems during exposure. Local signals reflect the actual head or eye posture. Remote signals represent the discordant position received by one system from the other system. †Head shift and visual shift aftereffects predicted for asymmetric exposure posture and discordance between head-trunk and eye-head systems.

displacement (i.e., an aftereffect) they would have to be turned in the displacement direction.

Of course, these recalibration predictions assume that the sensory signal from the remote discordant system has not undergone adaptation. To the extent that adaptation (postural or recalibration) occurs in either the eye-head or head-trunk system, the discordance stimulus for recalibration in the other system is reduced. Moreover, discordance signals from other sensorimotor systems may produce adaptation. For instance, Redding and Wallace $(1985,1987)$ argued that discordance between visual and auditory systems may provide a basis for recalibration aftereffects on the VS test. Thus, predictions can only be qualitative. Nevertheless, Table 1 clearly suggests that when adaptation is posturally based, HS should be greatest when subjects walk nearer the wall on the base side and VS should be greatest when subjects walk nearer the wall on the displacement side.

One other prediction is possible for this experimental situation. When subjects walk near the wall on the displacement side, the wall appears to be farther than it actually is, and they frequently bump the wall. These proprioceptive encounters provide discordance information and may prompt proprioceptive recalibration in the arm-trunk system (see Redding \& Wallace, 1985). If, as is likely, such arm shift (AS) is restricted to the arm on the wall side (i.e., no intermanual transfer; Redding \& Wallace, 1988), then a righthand PS test may include an AS component when the near wall is on the right side (i.e., on the side of the arm used in test), and PS may be expected to be larger in this condition than when the near wall is on the left side. In other conditions the PS test should be sensitive to HS, and these two tests should have similar results.
Finally, the usual total shift (TS) measure was included, requiring the subject to point at a visual target with his/her unseen hand. Comparison of the results for this test with the sum of VS + PS provided a check for conformity of the present conditions with the well-documented additivity of adaptive components (see Redding \& Wallace, 1987).

\section{METHOD}

\section{Subjects}

Right-handed undergraduate volunteers at Illinois State University served as subjects. All 64 subjects had self-reported normal vision or vision corrected to normal by contact lenses. Apparatus restrictions prevented testing of people who wore glasses or who were left-handed.

\section{Test Apparatus}

The test apparatus consisted of a wooden box $(122.0 \mathrm{~cm}$ wide $\times 75.0 \mathrm{~cm}$ high $\times 62.0 \mathrm{~cm}$ deep) placed on a table before an adjustable ophthalmological chair. Approximately the lower one-third ( $31.5 \mathrm{~cm}$ high $\times 112.0 \mathrm{~cm}$ wide) of the front surface of the box was covered by black felt material, with a sleeve for the subject's right arm, which prevented room light from entering the box. The remaining part of the front surface was covered by a plywood panel except for a rectangular opening $(5.5 \mathrm{~cm}$ high $\times 21.5 \mathrm{~cm}$ wide) centered $27.5 \mathrm{~cm}$ from the top of the box and $61.0 \mathrm{~cm}$ from each side, which permitted a view of the interior of the box. A masklike metal frame with an adjustable bite plate was suspended from an overhanging shelf at the top of the box. The subject wore welder's goggles with a Risley prism mounted in each eyepiece and stabilized his/her head within the frame by biting the plate, which was covered with dental registration material (vinyl polysiloxane $3 \mathrm{M}$ No. 6160 ). The frame was attached to the overhang with a rotary table (Oriel No. 1177) that permitted resistance-free rotation about the neck axis, and head position could be read to the nearest degree of arc from a scale located near the top of the frame. Curtains of black felt completely surrounded the subject's head, further prevent- 
ing room light from entering the box. A small speaker located approximately $10.0 \mathrm{~cm}$ directly above the subject's head emitted a .5 -sec tonal $(1000-\mathrm{Hz})$ signal, and a double-throw response lever was mounted on the left side of the chair at seat level.

Inside the box was a vertical curved surface with a radius of curvature of $57.3 \mathrm{~cm}$ from the subject's eyes; this surface covered the full lateral extent of the interior of the box. A horizontal row of red light-emitting diodes (LEDs) was mounted at eye level on this surface. Each LED had a diameter of $2.5 \mathrm{~mm}$, and they were mounted with $.5 \mathrm{~cm}\left(.5^{\circ}\right.$ of arc) between centers. Beginning about $1.0 \mathrm{~cm}$ below the LEDs and extending downward for $24.1 \mathrm{~cm}$, the curved surface was covered with black resistive paper (Western Union Teledeltos No. L-67). The subject wore a rubber tip on his/her right index finger, at the end of which was glued a small copper disk $(.75 \mathrm{~cm})$ with a thin wire that ran up the subject's arm and out of the box. When the subject touched the Teledeltos paper, lateral finger position could be read (to the nearest $.25^{\circ}$ of arc) remotely by the resistance change. A horizontal Plexiglas shelf between the LEDs and Teledeltos paper divided the box's interior and prevented the subject from touching the LEDs. A large button $(2.2 \mathrm{~cm}$ in diameter) located on the floor of the box directly below the subject's head permitted subjects to initiate pointing trials. All viewable surfaces of the box were painted flat black, and the box interior could be illuminated by two horizontal $(26.0 \times 1.4 \mathrm{~cm})$ fluorescent tubes (GE F6T5/CW) mounted on the interior surface of the front wall, each centered on a location approximately $27.0 \mathrm{~cm}$ to the side of and $13.0 \mathrm{~cm}$ below eye level.

All operations of the test box except the measures of head position were controlled by a microcomputer (Apple II+) with associated A/D converter (Applied Engineering 12-bit). Operations involved in measuring head position were performed by the experimenter and measurements were entered into the computer via the keyboard.

\section{Hallway}

The hallway was L-shaped. Each leg was about $29.1 \mathrm{~m}$ long; hallway width varied between 2.2 and $3.0 \mathrm{~m}$. One leg of the hallway was faced by solid walls, interrupted by doorway facings; one wall of the other leg was largely glass and opened onto a small patio. Noisy human traffic was common in the hallway, but subjects generally had no difficulty in negotiating the traffic.

\section{Design}

Sixteen subjects were tested in each of the four independent groups defined by factorial combination of displacement in the leftward or rightward directions and instructed walking on the left or right side of the hall. Before and after the hall exposure the subjects were given four kinds of tests in a partially counterbalanced order (a random Latin square). Assignment of subjects to these 16 conditions was alternated so that an equal number of subjects had been tested in each condition after every 16 subjects.

\section{Tests}

The VS test required each subject to remotely adjust a visual target to appear straight ahead of his/her nose. Each trial was initiated by a tonal signal and illumination of a single LED positioned randomly but equally frequently at $13^{\circ}, 14^{\circ}, 15^{\circ}, 16^{\circ}$ or $17^{\circ}$ in the left or right half of the visual field. Upon termination of the tone, the initial LED was turned off and the next LED toward the center of the field was turned on. This stepwise illumination was continued at a rate of one step per $.1 \mathrm{sec}$ (such that the initial LED appeared to move laterally across the field) until the subject judged the target to be located straight ahead of his/her nose, whereupon the subject quickly pulled up on the lever located at the side of the chair with his/her left hand. The leverpull extinguished the LEDs and immediately initiated the next trial.

For the PS test, each subject pointed sagittally straight ahead of his/her nose with the right hand and with vision occluded. Each trial was initiated by the subject's pressing the button on the floor of the box, which sounded the tonal signal. The subject then reached out and touched the resistive paper with the index finger of his/her right hand at a point the subject judged to be straight ahead of his/her nose. This touch produced an audible click from the speaker to signal successful contact. The subject then returned his/her hand to press the button and initiate the next trial.

The HS test required each subject to turn his/her head, without vision, to straight on the shoulders. For this test the head was free to rotate about the neck axis and the subject's eyes were closed. Each trial began with the experimenter's turning the subject's head to a random starting position (between $10^{\circ}$ and $20^{\circ}$ left or right). The subject was then instructed to turn his/her head to feel straight on his/her shoulders. Most commonly, the subject made one movement to a stop (i.e., without searching back and forth) before a reading was taken and the next trial was initiated.

The fourth test provided a measure of total shift (TS) between eye and hand by requiring subjects to point sagittally at an objectively straight-ahead target without sight of the right hand. The procedure here was identical with that for the PS test except that the LED objectively straight ahead of the subject's nose was illuminated and the subject was instructed to touch the resistive paper directly below this LED. The LED was illuminated at the end of the tonal signal and turned off when the subject touched the paper.

Ten measures were obtained on each of these tests before and after exposure; starting position for the VS and HS tests was random but equally frequently left and right of the body midline. Level of adaptation for each test was calculated by taking the difference between pretest and posttest means, with the adaptive direction of change being in the displacement direction for the VS and HS tests and opposite for the PS and TS tests.

Prior to each set of 10 pretest trials, 4 practice trials, which were not scored, were given. Starting position for these practice trials was randomly $13^{\circ}$ or $17^{\circ}$ left or right for the VS test and $10^{\circ}$ or $20^{\circ}$ left or right for the HS test. The interior of the box was illuminated only when instructions were being given prior to the practice trials for each test. At all other times the subjects were unable to see anything except the LEDs on VS and TS trials.

\section{Procedure}

Upon his/her arrival in the laboratory, each subject received a brief description of the experiment, including the fact that perceptualmotor coordination tests would be given before and after a 10-min period of walking in the hall while looking through prisms. Then a dental impression was taken, the goggles with the Risley prisms set to $0 \mathrm{D}$ (diopters) were fitted, the chair was adjusted, and pretest measures were obtained. Following the pretests, the Risley prisms were set to $30 \mathrm{D}\left(17.1^{\circ}\right)$ with the subject's eyes closed. The subject was then told to open his/her eyes and the experimenter guided the subject out into the hallway, positioning him/her on the left or right side of the hallway. The subject was instructed to walk along the left or right side of the hall in a normal manner, but not to look at his/her hands or feet. No subject was observed to violate this prohibition. The instruction to walk near the left or right wall was repeated if the subject strayed near the center of the hallway. While the subject was walking, the experimenter walked behind the subject, except for U-turns at the ends of the hallway, when the experimenter stood toward the center of the hallway, appearing in front of the subject when the turn was completed. Casual conversation was permitted. After $10 \mathrm{~min}$, the subject was returned to the testing chair and instructed to close his/her eyes while the prisms were reset to $0 \mathrm{D}$; the posttests were given without the subject's having seen the normal, undistorted world.

\section{RESULTS AND DISCUSSION}

Observation during exposure suggests that the majority of subjects (12 in each of the two groups) who received 
instructions to walk near the wall on the displacement side walked very near the wall, frequently encountering the wall with their arm or shoulder. The remaining 4 subjects in each group walked about in the middle of the halfhall on the displacement side. Of the subjects instructed to walk on the base side of the hall, most walked slightly to the base side of the hall center (14 subjects in the base left group and 15 in the base right group). The remaining subjects walked about in the middle of the half-hall on the base side. Thus, the manipulations seem to have had the desired result, although the subjects who walked nearest the wall on the base side seemed to compensate for the looming wall partially by their position in the hallway, rather than entirely by head posture.

Although the magnitude of head turning during exposure could not be reliably recorded, informal observation suggests that all subjects engaged in some (perhaps slight) head turning in the displacement direction during exposure. Thus, if anything, the eye posture basis for VS was less than maximal.

Mean level of adaptation and $95 \%$ confidence limits for each of the four tests are displayed in Table 2 for each of the four groups. Results for each of the four measures of adaptation and the test for additivity are presented separately.

\section{Head Shift}

Although none of the effects were very large, there were reliable differences in HS among the four groups. As predicted, HS was greater when the subjects were instructed to walk on the base side of the hall $[F(1,60)=$ $3.88, p=.05]$. This result suggests that even though the subjects partially compensated for the looming wall on the base side by positioning themselves toward the center of the hall, greater head turning in the displacement direction to fully see the hallway produced postural aftereffects. It seems likely that if compensation for the looming wall could be reduced by forcing subjects to walk nearer the wall on the base side, even larger posturally based HS would be found. Note that there is little evidence of prisminduced recalibration of head position. Even though the head posture indicated by the visual appearance of the hallway (i.e., "seen" head positions) was surely discordant with felt head position for all four groups, HS was smaller when less actual head nurning was expected (i.e., for groups instructed to walk on the displacement side of the hall).

\section{Visual Shift}

There appears to be no discernible relationship between VS and predictions based on expected eye posture. The only reliable finding was that VS was greater with leftward displacement $[F(1,60)=8.05, p<.01]$. This finding suggests that asymmetric eye posture is not a large source of VS in these data. There is even a tendency $[F(1,60)=2.64, p=.11]$ for VS to be largest for those conditions (i.e., walking near the base side) in which symmetric eye posture was expected (because of head turning) and smallest when asymmetric eye posture was expected. Note also that VS occurred even though all subjects showed some head turning in the displacement direction, which would have tended to produce symmetric eye postures.

Nor can the VS data be attributed to a failure of subjects to make head-relative judgments. If, during posttest, subjects compensated for a felt head position turned in the base direction by setting the target to straight ahead of the trunk (i.e., on the displacement side of straight ahead of the nose), the VS shift measure might be purely a measure of change in felt head position. This was clearly not the case. Comparison of the two measures showed that VS was larger than HS $[F(1,60)=6.96, p=.01]$ and that VS was affected by displacement direction, whereas HS was not $[F(1,60)=3.98, p<.05]$. Not only were the two measures different in magnitude, but more importantly, they were affected by different factors.

\section{Proprioceptive Shift}

The results for pointing straight ahead of the nose with the unseen right hand are well described by the assumptions that (1) this PS test includes any change in felt head position and (2) encounters with the wall by the arm and/or shoulder form the basis for discordance-based recalibration of felt arm position, which does not transfer between arms. As predicted, PS was reliable only for the condition in which subjects frequently bumped the wall

Table 2

Mean Level of Adaptation (in Degrees) and 95\% Confidence Limits for Head Shift, Visual Shift, Right-Hand Proprioceptive Shift, and Total Shift, as a Function of Nearest Hallway Wall Combined With Direction of Displacement

\begin{tabular}{|c|c|c|c|c|c|c|c|c|}
\hline \multirow[b]{3}{*}{ System } & \multicolumn{4}{|c|}{$\begin{array}{c}\text { Rightward Displacement } \\
\text { (Base Left Prisms) }\end{array}$} & \multicolumn{4}{|c|}{$\begin{array}{l}\text { Leftward Displacement } \\
\text { (Base Right Prisms) }\end{array}$} \\
\hline & \multicolumn{2}{|c|}{ Right Wall } & \multicolumn{2}{|c|}{ Left Wall } & \multicolumn{2}{|c|}{ Right Wall } & \multicolumn{2}{|c|}{ Left Wall } \\
\hline & $M$ & $\mathrm{CL}$ & $M$ & $\mathrm{CL}$ & $\boldsymbol{M}$ & $\mathrm{CL}$ & $M$ & $\mathrm{CL}$ \\
\hline Head-Trunk (HS) & 0.1 & 1.2 & 1.1 & 1.3 & 1.2 & 1.3 & -0.2 & 1.2 \\
\hline Eye-Head (VS) & 0.5 & 1.2 & 1.2 & 1.2 & 3.0 & 1.0 & 1.9 & 1.2 \\
\hline Arm-Head (PS) & 2.2 & 1.8 & 0.7 & 1.6 & 0.8 & 2.1 & -0.3 & 2.4 \\
\hline Eye-Hand (TS) & 2.7 & 1.0 & 2.2 & 1.4 & 3.3 & 1.0 & 2.2 & 1.1 \\
\hline Eye-Hand (VS+PS) & 2.7 & 1.7 & 1.9 & 2.2 & 3.8 & 2.1 & 1.6 & 2.6 \\
\hline
\end{tabular}

Note-CL = confidence limits, HS = head shift, VS = visual shift, PS = proprioceptive shift, TS = total shift. The data are grouped by sensorimotor system to facilitate comparison with the predictions in Table 1 . 
with their right arm and/or shoulder. For the remaining conditions, PS and HS were similar in magnitude, and direct comparison of PS and HS produced an $F$ ratio greater than 1.00 only for subjects with rightward displacement who walked near the right wall $[F(1,15)=$ $2.49, p=.13]$.

\section{Additivity Test}

Consistent with previous studies, these data show almost perfect additivity. The sum of VS +PS (2.7) is not significantly different from TS $(2.8)[F(1,60)<1.00]$, and the only source of variance in this analysis with an $F$ ratio greater than 1.00 is the main effect for nearest wall. Both measures show a slight tendency toward greater values when subjects walked near the right wall $[F(1,60)$ $=3.24, p=.07]$. Thus, there is no evidence that the present results violate the basic assumption of a linear system.

\section{CONCLUSIONS}

Changes in felt head position during hallway exposure to displacing prisms appear to be a postural aftereffect of head turning in the displacement direction, rather than a discordance-reduction recalibration effect. The magnitude of HS was larger for conditions in which subjects were expected to make larger head turnings to avoid the looming wall and see the entire hallway (i.e., when the nearest wall was on the base side), even though discordance between seen and felt head position was arguably the same for all conditions. Nevertheless, such posturally based HS was not very reliable in the present study and may not be a large component of the adaptive response to prism exposure in hallway situations.

Visual adaptation during hallway exposure does not seem to be explicable in terms of either postural aftereffects of asymmetric eye posture or contamination of the VS measure by HS through a failure of subjects to make head-relative judgments. The magnitude of VS tended to be largest for those conditions in which symmetric eye posture was expected because of head turning in the displacement direction (i.e., when the subject was walking near the base side wall), and smallest when asymmetric eye posture was expected. Although the magnitudes of HS and VS were comparable with rightward displacement, the large VS obtained with leftward displacement was not comparable with the small HS found under these conditions.

These results suggest that the basis of VS may reside in recalibration of the eye-head system prompted by discordant signals from the head-trunk and/or other sensorimotor systems. For instance, Redding and Wallace (1985, 1987) argued that in hall exposure, visible sound sources provide opportunities for auditorily guided visual locational response, with consequent registration of discordance and perceptual recalibration in the visual (eye-head) system. The present findings are consistent with this directionality-of-guidance model (Redding et al., 1985), and variability in the occurrence of visible sound sources may explain the differences in VS among the four groups.

The finding that PS was reliable only under conditions in which subjects frequently encountered the wall with their right arm and/or shoulder (i.e., when they were walking near the wall on the displacement side) is also consistent with the directionality-of-guidance model. Subjects first see the wall as farther away than it really is, anticipate encountering the wall with their arm and/or shoulder, and when the wall is encountered sooner than expected the discordance between seen and felt wall position is attributed to error in the proprioceptive (armhead) system, with consequent perceptual recalibration in the arm-head system. Thus, large PS can be expected in hall exposure when conditions are such as to produce frequent proprioceptive encounters with visible obstacles or when conditions produce large asymmetric head postures.

\section{REFERENCES}

Cohen, M. M. (1967). Continuous versus terminal visual feedback in prism aftereffects. Perceptual \& Motor Skills, 24, 1295-1302.

Craske, B., Crawshaw, M. (1974). Adaptive changes of opposite sign in the oculomotor systems of the two eyes. Quarterly Journal of Experimental Psychology, 26, 106-113.

Craske, B., Crawshaw, M. (1978). Spatial discordance is a sufficient condition for oculomotor adaptation to prisms: Eye muscle potentiation need not be a factor. Perception \& Psychophysics, 23, 75-79.

EBenHoltz, S. M. (1976). Additivity of aftereffects of maintained head and eye rotations: An alternative to recalibration. Perception \& Psychophysics, 19, 113-116.

Hamilton, C. R. (1964). Intermanual transfer of adaptation to prisms. American Journal of Psychology, 77, 457-462.

HARRis, C. S. (1965). Perceptual adaptation to inverted, reversed, and displaced vision. Psychological Review, 72, 419-444.

HAY, J. C., \& PiCK, H. L., JR. (1966). Visual and proprioceptive adaptation to optical displacement of the visual stimulus. Journal of Experimental Psychology, 71, 150-158.

HowARD, I. P., \& ANSTIS, T. (1974). Muscular and joint-receptor components in postural persistence. Joumal of Experimental Psychology, 103, 167-170.

KoHLER, I. (1964). The formation and transformation of the perceptual world. Psychological Issues, 3(Whole No. 4), 1-173.

Redding, G. M., Clark, S. E., \& Wallace, B. (1985). Attention and prism adaptation. Cognitive Psychology, 17, 1-25.

Redding, G. M., \& Wallace, B. (1976). Components of displacement adaptation in acquisition and decay as a function of hand and hall exposure. Perception \& Psychophysics, 20, 453-459.

Redding, G. M., \& Wallace, B. (1978). Sources of "overadditivity" in prism adaptation. Perception \& Psychophysics, 24, 58-62.

Redding, G. M., \& Wallace, B. (1985). Perceptual-motor coordination and adaptation during locomotion: Determinants of prism adaptation in hall exposure. Perception \& Psychophysics, 38, 320-330.

Redding, G. M., \& WALLACE, B. (1987). Perceptual-motor coordination and prism adaptation during locomotion: $A$ control for head posture contributions. Perception \& Psychophysics, 42, 269-274.

Redding, G. M., \& Wallace, B. (1988). Components of prism adaptation in terminal and concurrent exposure: Organization of the eyehand coordination loop. Perception \& Psychophysics, 44, 59-68.

WAllace, B., RedDing, G. M. (1979). Additivity in prism adaptation as manifested in intermanual and interocular transfer. Perception \& Psychophysics, 25, 133-136. 
Welch, R. B. (1978). Perceptual modification. New York: Academic Press.

WELCH, R. B., \& WARREN, D. H. (1980). Immediate perceptual response to intersensory discrepancy. Psychological Bulletin, 88, 638-667. Wilkinson, D. A. (1971). Visual-motor control loop: A linear system? Journal of Experimental Psychology, 89, 250-257.

\section{NOTES}

1. The term recalibration is reserved for calibration processes that determine the deviation of one sensorimotor system (e.g., eye-head) from the (extemal) standard provided by another (discordant) system so as to ascertain the proper correction factors. In the visual system, such perceptual recalibration could consist in a change in either retinal local sign or registered eye position. Current theory does not permit a test between these two possible accounts of visual change, and the present research is neutral with respect to this problem. Muscie potentiation effects do not involve any reference to an external standard, but simply arise from a shift in a muscle group's resting position produced by atypical exercise. Both recalibration and postural aftereffects have perceptual consequences, and both are assumed to be localized within a sensorimotor system, but the underlying mechanisms are different in nature. Recalibration requires input from an external discordant system, whereas postural aftereffects do not.

2. An immediate effect of discordance is that the head feels the way it looks to be, turned in the base direction. However, there is no evidence that such visual capture is related to adaptation (e.g., Welch \& Warren, 1980), and the local signal for head position is assumed to be available for comparison with the discordant visual information.

(Manuscript received September 3, 1987; revision accepted for publication December $7,1987$.

\title{
Announcement
}

\author{
International Conference on Cognitive Neuropsychology \\ Harrogate, England \\ July 20-23, 1989
}

The International Conference on Cognitive Neuropsychology, sponsored by the British Psychological Society Cognitive Section, will be held in the Cairn Hotel, Harrogate, England, on July 20-23, 1989.

Submissions for papers on the following topics are invited: memory, language disorders, reading and writing disorders, visual recognition, attention, action and movement, childhood disorders, and remediation. There will be no symposia. The deadline for submissions is February 1989.

For additional information, contact John Beech or Ann Colley, Conference Organizers, Department of Psychology, The University of Leicester, Leicester LE1 7RH, England. 